\title{
MONITOREO DE EMOCIONES BÁSICAS AUTOREPORTADAS RELACIONADAS CON EL CONFINAMIENTO POR COVID-19 Y EL REGRESO A LA VIDA COTIDIANA
}

\author{
MONITORING SELF-REPORTED BASIC EMOTIONS RELATED TO COVID-19 \\ CONFINEMENT AND RETURN TO EVERYDAY LIFE
}

Eloy Maya Pérez ${ }^{1}$

Marcela Ortega ${ }^{2}$

Jonathan Alejandro Galindo Soto ${ }^{3}$

Aceptado: $25 / 11 / 2020$

Publicado online: $14 / 12 / 2020$

\begin{abstract}
RESUMEN
Las emociones son un elemento central para comprender de la experiencia humana. Con la intención de abonar a tal comprensión, se diseñó el presente estudio orientado al análisis de las emociones básicas relacionadas con el confinamiento por covid-19 y con el regreso a la vida cotidiana. Para lo cual se elaboró una encuesta con 21 ítems, participaron 230 personas, de los cuales el $73.2 \%$ fueron mujeres y $26.4 \%$ hombres, con edades que oscilaron entre los 22 y los 71 años, la característica central para la elección de dichos participantes fue que se encontrarán laborando antes y en los meses posteriores a la declaración de confinamiento en México. Los resultados describen la existencia de emociones de carácter negativo asociadas con la autopercepción de los participantes; especialmente el miedo como la emoción que más se manifestó ( $f=117$ ), seguido de la tristeza y el enojo. Se concluye que en relación con el principio psicológico de adición emocional existe una tendencia negativa hacia los afectos vividos.
\end{abstract}

Palabras clave: Emociones, confinamiento, vida cotidiana, coronavirus

\begin{abstract}
Emotions are a central element for understanding the human experience. With the intention of paying for this understanding, the present study was designed aimed at the analysis of the basic emotions related to confinement by covid-19 and with the return to daily life. For which a survey with 21 items was developed, 230 people participated, of which $73.2 \%$ were women and $26.4 \%$ men, with ages ranging between 22 and 71 years, the central characteristic for the choice of said participants was who will be working before and in the months after the declaration of confinement in Mexico. The results describe the existence
\end{abstract}

\footnotetext{
1 Licenciatura en Psicología Clínica, Departamento de Enfermería Clínica, Campus Celaya-Salvatierra de la Universidad de Guanajuato, México. (D)0000-0001-6195-5917 Corresponsal: Av. Ing. Barros Sierra No. 201 Ejido de Santa María del Refugio C.P. 38140 Celaya, Guanajuato, México, Teléfono: 5201 (461) 598 5922, Celular: (+052) 461401073, email: e.maya@ugto.mx. 2 Licenciatura en Enfermería Clínica, Departamento de Enfermería Clínica, Campus Celaya-Salvatierra de la Universidad de Guanajuato, México. ormaii@hotmail.com. (D)0000-0002-0682-0283

3 Licenciatura en Psicología Clínica, Departamento de Enfermería Clínica, Campus Celaya-Salvatierra de la Universidad de Guanajuato, México. jonathan.galindo@ugto.mx. (D) 0000-0001-6351-8681
} 
of negative emotions associated with the participants' self-perception; especially fear as the emotion that manifested the most $(f=117)$, followed by sadness and anger. It is concluded that in relation to the psychological principle of emotional addition there is a negative tendency towards experienced affects.

Keywords: Emotions, home confinement, daily life, coronavirus.

\section{INTRODUCCIÓN}

En marzo del 2020, tras una severa evaluación de los riesgos en salud a los que estaba expuesta la población mundial la Organización Mundial de la Salud decidió considerar al nuevo coronavirus SARS-Cov-2 como la causa de la pandemia por COVID-19, por lo que se comenzó a vivir una situación excepcional por el avance de la pandemia por el coronavirus 2 del síndrome respiratorio agudo grave y la inédita medida de aislamiento social preventivo y obligatorio utilizada por varios países (Ribot, Chang \& González, 2020), situación que ha tenido un impacto psicosocial considerable, mismo que ha despertado un interés repentino en la comunidad académica dedicada al estudio de la salud mental.

Considerando que las alteraciones a la salud constituyen un problema que va más allá de las intervenciones clínicas pues como afirman Thielmann y Illnait (2012) una crisis en salud impacta de forma negativa sobre la vida cotidiana, sin embargo, en el caso de la salud mental ante el brote de COVID-19 se cuenta con poca evidencia disponible que revise las alteraciones a la salud mental y el bienestar psicosocial de la población; Urzúa, Vera, Caqueo y Polanco (2020) afirman que ya algunos especialistas reportan una serie de reacciones y estados psicológicos observados en la población incluyendo efectos en la salud mental, o bien se experimentan condiciones severas de estrés como afirma la OMS (2020); Ojeda y De Cosío (2020), afirman que existen factores de estrés específicos del brote de COVID-19, entre los cuales se incluye: el riesgo de estar infectado e infectar a otros; los síntomas de otros problemas de salud pueden confundirse con el COVID-19; los cuidadores pueden sentirse cada vez más preocupados porque sus hijos estén solos debido al cierre de las escuelas y por el riesgo del deterioro de la salud física y mental de personas vulnerables. Håkansson, Fernández, Menchón, Potenza y Jiménez (2020) aseguran que la pandemia de COVID-19 tiene el potencial de empeorar los problemas de salud mental en la población en general, incluido el aumento de la participación en conductas adictivas.

Otro grupo importante de investigaciones sobre el COVID-19 y la salud mental se orientó al personal de salud, revisando el impacto psicológico a nivel de estrés, ansiedad, síntomas depresivos, insomnio, negación, ira y temor en el personal de salud que atiende directamente a personas infectadas condición que les ha provocado sentir frustración, sufrir discriminación, vivir en el aislamiento e interactuar con pacientes con emociones negativas además de la pérdida del contacto con la familia y al agotamiento físico y mental (Lozano, 2020). Lai et al. (2020) analizaron la salud mental de médicos y enfermeros de primera línea de atención de hospitales de China, y reportan que un alto porcentaje reconoció sentirse angustiado, con síntomas de depresión, ansiedad e insomnio, por su parte Rossi et al. (2020), realizaron un estudio entre personal de salud en Italia, donde lograron determinar una alta presencia de estrés post-traumático, depresión e insomnio con mayor incidencia en mujeres.

En relación con las emociones y la vida afectiva durante la pandemia, De la Serna (2020) afirma que una situación como la que experimenta el mundo desde inicios de este año suscita estados de estrés que trascenderán en cada persona en virtud de sus propias características psicológicas, por ejemplo, se estima que para algunas personas las consecuencias estresantes ocurrirán a medio y largo plazo una vez superada la cuarentena. En este sentido, Muñoz, Molina, Ochoa, Sánchez y Esquivel (2020) 
reflexionan en relación con los elementos que deben tomarse en cuenta para evaluar la respuesta psíquica ante COVID-19 están los factores sociodemográficos, las demandas sociales y laborales, la estructura de personalidad, las estrategias de afrontamiento empleadas y las redes de apoyo social con que cuenta cada individuo.

En un estudio reciente relacionado con la salud mental y el covid-19, Martínez (2020) planteó que las pandemias suelen traer consigo la necesidad imperiosa de lidiar con sus manifestaciones y secuelas, las cuales suelen estar inmersas en incertidumbre y ponen de manifiesto la fragilidad de nuestro entorno y la vida. Johnson et al. (2020) reportan a través de una investigación realizada en Argentina que los sentimientos a lo que más se refirieron los participantes fueron el miedo incertidumbre y preocupación. Balluerka et al. (2020) realizaron un estudio con la intención de explorar el grado de afectación psicológica en la población española destacando la presencia de ansiedad y miedo, es decir, una considerable presencia de incertidumbre especialmente por la preocupación por padecer o contraer la enfermedad covid-19 u otras relacionadas.

Los estudios contemporáneos sobre las emociones han determinado que están constituidas por cinco componentes: expresión motora, componente cognitivo, componente neurofisiológico, componente motivacional y la experiencia subjetiva (Cossini, Rubinstein \& Politis, 2017). El presente estudio retoma la propuesta de Paul Ekman (1971) en la que describe la presencia de seis emociones básicas en todos los seres humanos: alegría, tristeza, enojo, sorpresa, miedo y rechazo. Matsumo, Pérez y López (2013) comentan que diversos estudios relacionados con la comprensión de las emociones determinan su universalidad. Se retoma tal propuesta considerando las aportaciones de Ekman y Cordaro (2011) en las que afirman que las emociones básicas son respuestas que surgen de manera inmediata ante la estimulación del medio y se caracterizan por ser innatas, universales, de procesamiento automático y sin influencia sociocultural. Finalmente es importante destacar que dentro de los modelos de análisis de las emociones se recurre al principio psicológico conductual de adición descrito por Carrillo et al. (2006), en el que se explica que cuando al experimentar múltiples emociones en un mismo momento, las emociones se suman para obtener una guía de los que se está vivenciando emocionalmente, es decir, sea posible reconocer como emociones básicas a lo positivo y/o negativo.

\section{OBJETIVOS}

- Identificar las emociones básicas autoreportadas relacionadas con el confinamiento por covid19 y con el regreso a la vida cotidiana.

- Describir la frecuencia de emociones autoreportadas relacionadas con el confinamiento por covid-19 y el regreso a la vida cotidiana.

\section{METODOLOGÍA}

Se realizó un estudio descriptivo de corte transversal con la intención de conocer las emociones básicas autoreportadas por los participantes relacionados con el confinamiento y ante el posible retorno a las actividades de la vida cotidiana post confinamiento por coronavirus covid-19. Para alcanzar este propósito, se diseñó una encuesta de 21 ítems, divida en tres secciones: 1 . Datos generales de los participantes, 2. Monitoreo de las emociones básicas que contenía ítems como: ¿Cuáles fueron tus reacciones emotivas al enterarte de la existencia del covid-19?, ¿Cuáles fueron tus reacciones emotivas cuando te dieron la noticia en tu empleo sobre el inicio del confinamiento?, ¿Cuáles fueron tus reacciones emotivas al comenzar a pasar más tiempo en casa?, ¿Cuáles fueron tus reacciones emotivas al pasar más tiempo con tu familia en casa?, ¿Cuáles fueron tus reacciones emotivas al tener que adecuar los hábitos alimenticios a una nueva dinámica en casa?, ¿Cuáles fueron tus reacciones emotivas en relación con el cuidado de tu salud durante el confinamiento?, y 
3. Reacciones emotivas con ítems como: ¿Cuál de estas emociones representa lo que sientes en relación con el fin del aislamiento?, El regreso a tus actividades laborales o actividades de la vida cotidiana te provoca, ¿Cuál de estas emociones representa lo que sientes en relación con el cuidado de tu salud a partir del regreso a tus actividades laborales o actividades de la vida cotidiana?, ¿Con qué emoción representas la reinserción a la vida cotidiana tras el fin del confinamiento por coronavirus?.

El instrumento se orientó en la propuesta de Paul Eckman (1978) sobre las seis emociones básicas, dentro de los criterios de inclusión se consideró que los participantes fueran personas que se encontrarán realizando actividades laborales remuneradas antes de la declaración de pandemia y que hayan sido enviadas a trabajar a su casa a partir de que se decretó la emergencia sanitaria. El total de la muestra estuvo conformada por 230 participantes, de los cuales el $73.2 \%$ fueron mujeres y $26.4 \%$ hombres con edades que oscilan entre los 22 y los 71 años de edad ( $M=34.3$ años, $D E=9$ ); de ellos, el $47.6 \%$ mencionó ser soltero, mientras que el 31.6\% está casado, el 15.2\% viven en unión libre, divorciados $4.3 \%$ y viudos $0.9 \%$. En relación con el nivel de estudios, la distribución fue la siguiente: $37.2 \%$ pertenecen a licenciatura, $34.2 \%$ maestría, $21.6 \%$ doctorado, $6.1 \%$ bachillerato y $0.4 \%$ educación básica.

\section{RESULTADOS}

En relación con las seis emociones básicas y el confinamiento, los participantes comentaron que han percibido emociones de carácter negativo y positivo, ambas con una intensidad considerable. En primera instancia, se retomó el criterio de suma de emociones clasificándolas de acuerdo con las propiedades afectivas de las mismas y, posteriormente, se organizaron los datos en dos conjuntos de emociones negativas y positivas. Para ello, se tomó como criterio la presencia de dos o más emociones para poder agruparlas y determinar con base en la frecuencia de la respuesta.

En el primer conjunto se destacan las emociones negativas, compuestas por el miedo, la sorpresa, la tristeza y el rechazo en ese orden. El miedo aparece como la emoción más representativa de lo que el confinamiento ha provocado. En la tabla 1 se observa que el miedo, conceptualizado como la sensación de riesgo o daño (Eckman, 2003) y está apoyado en el desconocimiento de la situación y lo que ocurrirá con relación a esta, aparece con una mayor frecuencia $(f=117)$ en relación con el resto de las emociones reportadas por los participantes y la tristeza -cuyo desencadenante universal está puesto sobre la pérdida y la ausencia (Eckman, 2003) o como señala Christlieb (1994) representa todo lo que ha quedado indefinidamente interrumpido con la ausencia- aparece como una emoción negativa $(f=96)$ presente en los participantes. Dentro de este mismo conjunto de emociones negativas el rechazo como emoción básica, es autopercibido como neutral $(f=100)$ así como también el enojo $(f=97)$.

Tabla 1

Frecuencia de las emociones básicas autopercibidas.

\begin{tabular}{lccc} 
& Mayor & $f$ & \\
& Neutral & Menor \\
\hline Rechazo & 38 & 100 & 92 \\
Miedo & 117 & 80 & 33 \\
Enojo & 64 & 97 & 69 \\
Alegría & 81 & 111 & 38 \\
Sorpresa & 100 & 94 & 36 \\
Tristeza & 96 & 74 & 60 \\
\hline
\end{tabular}

Como emociones positivas, se destaca la frecuencia de la sorpresa $(f=100)$ que de acuerdo con Eckman (1973) es posible considerarla como una emoción que anticipa el miedo, pero también 
asociada con la expectativa y la felicidad por las noticias recibidas. Es de llamar la atención que la alegría es mayormente percibida $(f=111)$ con la categoría neutral, considerando que representa la respuesta emotiva asociada con lo positivo, específicamente se caracteriza por ser una respuesta emocionalmente densa que puede arrastrarnos a un estado de ánimo eufórico o subido (Eckman, 2003).

La dispersión de las emociones básicas muestra que la tendencia en la elección es hacia las emociones negativas (ver Figura 1). En cuanto al miedo, aparece con el 61.30\% de autoreportes que constituyen la mayor concentración, indicador que llama la atención sobre la salud mental de los participantes.

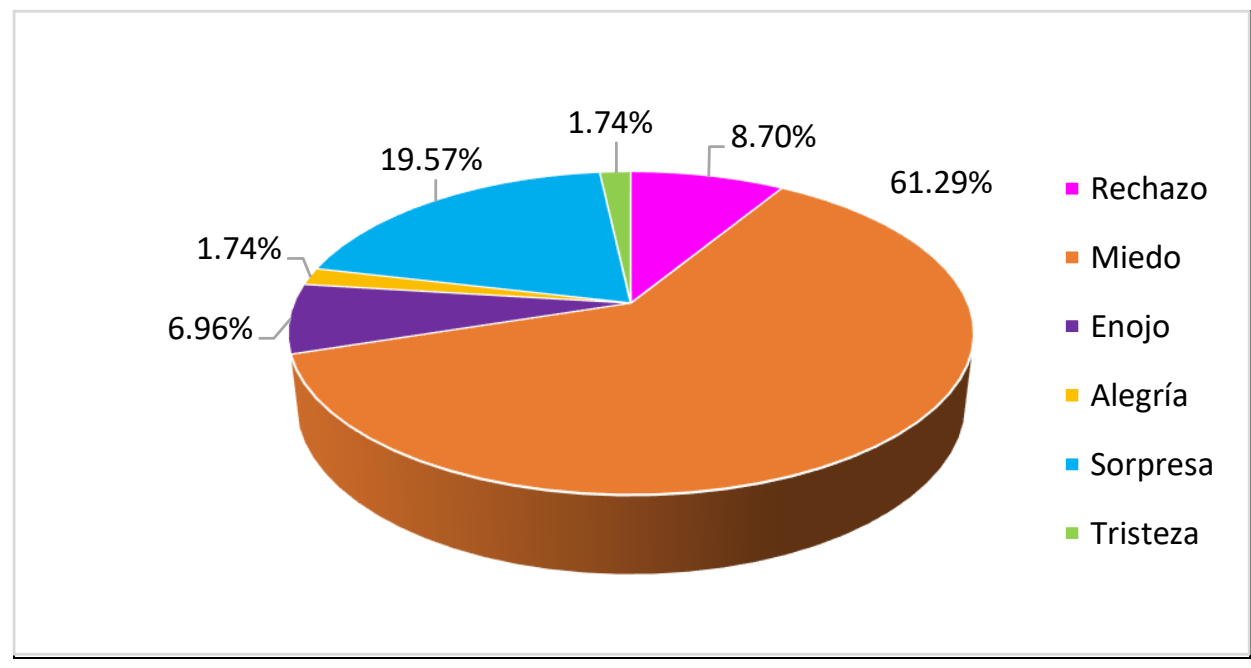

Figura 1

Dispersión de emociones básicas autoreportadas en relación con la etapa de confinamiento

La segunda parte del instrumento se dedicó a medir las emociones autopercibidas frente al regreso a la vida cotidiana; de éste se seleccionaron cuatro ítems que muestran reacciones emocionales vinculadas con el regreso a las actividades laborales o actividades de la vida cotidiana a través del fin del aislamiento; el cuidado de tu salud y la reinserción laboral.

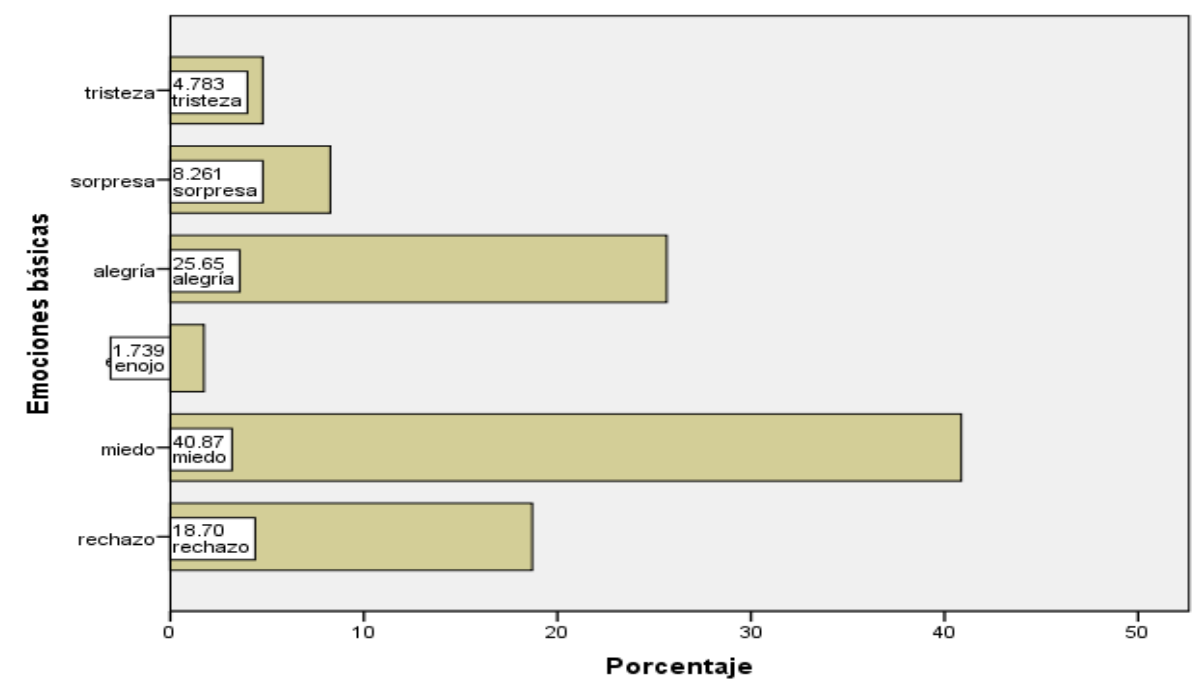

Figura 2

Distribución porcentual de emociones básicas autoreportadas 
En la Figura 2, se muestra la presencia de las emociones básicas autopercibidas donde el miedo se presenta nuevamente como la más representativa (40.8\%), considerándose en palabras de Bolaños (2016) como un autocoacción relacionada con el riesgo, posiblemente esté orientado en el temor al contagio de sí mismo o alguno de los familiares por motivos de la reinserción social. También llama la atención el porcentaje que se le otorga a la alegría que contrasta con lo analizado en el apartado anterior, pues se ubica porcentualmente (25.6\%) como la segunda emoción autopercibida por los participantes, los significados de esta elección podrán variar, pero se puede interpretar que por lo general están vinculados con el propio fin del confinamiento (De la Serna, 2020). El rechazo (18.7\%) -que de acuerdo con Eckman (2003) es posible comprenderla como una respuesta de aversión o repudio- contrasta con la presencia de la alegría, sin embargo, con lo que se reafirma la autopercepción desde una postura negativa de las emociones.

Tabla 2

Frecuencia de las emociones básicas autopercibidas en relación con el regreso a actividades laborales y el cuidado de la salud

\begin{tabular}{lcc}
\hline & & $f$ \\
& Regreso a actividades laborales & Cuidado de la salud \\
\hline Rechazo & 48 & 24 \\
Miedo & 100 & 130 \\
Enojo & 3 & 6 \\
Alegría & 61 & 41 \\
Sorpresa & 13 & 24 \\
Tristeza & 5 & 5 \\
\hline
\end{tabular}

Para finalizar el análisis, es importante señalar que en relación con los temas de "Regreso a actividades laborales" y "Cuidado de la Salud", mostradas en la tabla 2, la percepción reportada coincide con la tendencia emocional anteriormente descrita, pues se muestra que el miedo como emoción básica autopercibida ( $f=100$ y 130 respectivamente) estuvo presente en la información que compartieron.

\section{DISCUSIÓN}

La investigación realizada da cuenta de la experiencia emocional durante confinamiento por covid19 y con el regreso a la vida cotidiana, para ambas situaciones se reconoce que existe impacto en la salud mental de los participantes y este es expresado en emociones de carácter negativo, principalmente, se evidencia el miedo, la tristeza y el enojo, generadores de incertidumbre y como afirma Johnson et al. (2020), son propios de un sentido de ruptura con la cotidianidad creado durante el periodo de aislamiento.

Después de analizar los datos recolectados y vinculándolos con el modelo el principio conductual de adición de las emociones de Staats et al. (1996), en el que se describe que las connotaciones afectivas positivas y negativas provienen tanto de los pensamientos como de las experiencias de la vida real -sobre todo, que tales experiencias determinan la vivencia impactando en la salud mental de la población-, se destaca la tendencia negativa de las emociones autopercibidas. Atendiendo al método descrito por Carrillo et al. (2006), en el que se considera que, si las personas experimentan múltiples fuentes de estimulación emocional, las emociones se suman destacando no solo la frecuencia de aparición de una u otra sino la intensidad con que son percibidas, relatas o descritas.

En este caso, durante la exposición de los resultados fue claro ver la presencia del rechazo y, sobre todo, del miedo en mayor medida que otras emociones básicas como la alegría. Lo cual nos orienta a reflexionar sobre la presencia de emociones complejas, definidas por Tabernero \& Politis (2014) como aquéllas que surgen de la combinación de emociones primarias, o bien derivadas de las 
básicas, generalmente por combinación entre ellas como afirma Bisquerra (2010) como la incertidumbre, entendida como un estresor potente de acuerdo con Tabernero et al. (2014) o bien una es una amenaza por evitar (Szeto \& Sorrentino, 2009).

Es importante destacar que los estados emocionales negativos están asociados con la aversión, la defensa, el escape, la evitación y otras conductas de protección o que pueden generar emociones orientadas a la adaptación individual a los sucesos estresantes. El miedo, por ejemplo, se presentó una constante en las actitudes hacia lo que se está viviendo durante este periodo de confinamiento por covid-19, y resulta congruente poder experimentarlo durante el regreso a la vida cotidiana, provocando, quizá, que la alteración de estilos de vidas, hábitos y rutinas que generen procesos emocionales de mayor impacto como la incertidumbre, que se relaciona con aquello que está fuera de la comprensión inmediata y del control de la persona. Useche (2008) afirma que el miedo se asocia con los fenómenos que fracturan la confianza sobre la que puede construirse la sociabilidad; pensar en que el miedo se transforma en incertidumbre supone una idea de complejidad emocional a la que nos enfrentamos en este periodo de contingencia sanitaria; la incertidumbre de acuerdo con De Sousa (2016), es la vivencia de las posibilidades que surgen de las múltiples relaciones que pueden existir, en este sentido, por las dificultades y el riesgo en salud que representa.

Es importante reconocer los posibles caminos que puede orientar una investigación de este tipo en la que se revisen otros aspectos afectivos como por ejemplo las emociones positivas que el encierro suscitó con la familia, o bien en relación con la creación de estrategias autocuidado en salud.

\section{REFERENCIAS BIBLIOGRÁFICAS}

Balluerka, N., Gómez, J., Hidalgo, M., Gorostiaga, A., Espada, J., Padilla, J, \& Santed, M. (2020). Las consecuencias psicológicas de la covid-19 y el confinamiento. Informe de investigación. https://www.ub.edu/web/ub/ca/menu eines/noticies/docs/Consecuencias psicologicas COVID19.pdf

Bisquerra, R. (2010). Psicopedagogía de las emociones. Editorial Síntesis

Cossini, C., Rubinstein, Y. \& Politis, G. (2017). ¿Cuántas son las emociones básicas? Estudio preliminar en una muestra de adultos mayores sanos. Anuario de Investigaciones, 24, 253-257. https://www.redalyc.org/pdf/3691/369155966032.pdf

De la Serna, J. (2020). Aspectos Psicológicos del COVID-19. Tektime. https://www.researchgate.net/publication/340536973 Aspectos Psicologicos del COVID-19

De Sousa. B. (2016). La incertidumbre, entre el miedo y la esperanza. En: Trotta, N. \& Gentil, P. (comp.). América Latina: La democracia en la encrucijada. CLACSO.

Ekman, P. (1971). Constants across cultures in the face and emotion. Journal of Personality and Social Psychology, $17(2)$,

http://www.communicationcache.com/uploads/1/0/8/8/10887248/constants across cultures in th e face and emotion.pdf

Ekman, P. \& Cordaro (2011) What is Meant by Calling Emotions Basic. Emotions review, 3 (4), 364-370. https://journals.sagepub.com/doi/abs/10.1177/1754073911410740?journalCode=emra

Håkansson, A., Fernández, F., Menchón, J., Potenza, M., \& Jiménez, S. (2020). Gambling During the COVID-19 Crisis - A Cause for Concern. Journal of addiction medicine, 14(4), 10-12. https://doi.org/10.1097/ADM.0000000000000690

Johnson, M., Saletti, L. \& Tumas, N. (2020). Emociones, preocupaciones y reflexiones frente a la pandemia del COVID-19 en Argentina. Ciência \& Saúde Coletiva, 25(1), 2447-2456. https://doi.org/10.1590/141381232020256.1 .10472020

Lai, M., Ma, S., Wang, Y., Cai, Z., Hu, J., Wei, N. Wu, J., Du, H., Chen, T., Li, R., Tan, H., Kang, S., Wang, Y., Yao, L., Huang, M., Wang, H., Wang, G, Liu, Z., \& Hu, S. (2020). Factors associated with mental health outcomes among health care workers exposed to coronavirus disease 2019. JAMA Newtwork Open, 3(3), https://doi.org/10.1001/iamanetworkopen.2020.3976 
Leperski, K. (2017). El paradigma de las emociones básicas y su investigación. Hacia la construcción de una crítica (conferencia). IX Congreso Internacional de Investigación y Práctica Profesional en Psicología XXIV Jornadas de Investigación XIII Encuentro de Investigadores en Psicología del MERCOSUR. Facultad de Psicología. Universidad de Buenos Aires. https://www.aacademica.org/000-067/146.pdf

Lozano, A. (2020). Impacto de la epidemia del Coronavirus (COVID-19) en la salud mental del personal de salud y en la población general de China. Revista Neuropsiquiatría, 83(1), 51-56. https://doi.org/10.20453/rnp.v83i1.3687

Martínez, A. (2020). Pandemias, COVID-19 y Salud Mental: ¿Qué Sabemos Actualmente? Revista Caribeña de Psicología, 4(2), 143-152. https://doi.org/10.37226/rcp.v4i2.4907

Matsumo, D., Pérez, M. \& López, R. (2013). Lectura de la expresión facial de las emociones: investigación básica en la mejora del reconocimiento de emociones. Ansiedad y estrés, 19(2-3), 121-129. https://www.researchgate.net/publication/258119111 Lectura de la Expresion Facial de las Em ociones Investigacion basica en la mejora del reconocimiento de emociones

Muñoz, S., Molina, D., Ochoa, R., Sánchez, O. \& Esquivel, J. (2020). Estrés, respuestas emocionales, factores de riesgo, psicopatología y manejo del personal de salud durante la pandemia por COVID-19. Acta Pediátrica Mexicana, 41(1), 127-S136. https://www.medigraphic.com/pdfs/actpedmex/apm2020/apms201q.pdf

Ojeda, H. \& De Cosío, F. (2020). COVID-19 y salud mental: mensajes clave. OPS. https://www.researchgate.net/publication/340805424 COVID-19 y salud mental mensajes clave

Organización Mundial de la Salud (12 de marzo del 2020). Consideraciones psicosociales y de salud mental durante el brote de COVID-19. https://www.paho.org/sites/default/files/2020-03/smaps-coronaviruses-final-17-mar-20.pdf

Ribot, V., Chang, N. \& González, A. (2020). Efectos de la COVID-19 en la salud mental de la población. Revista Habanera de Ciencias Médicas,

19.

http://www.revhabanera.sld.cu/index.php/rhab/article/view/3307

Rossi, R., Socci, V., Pacitti, F., DiLorenzo, G., Marco, A., Siracusano, A., \& Rossi, A. (2020). Mental health outcomes among frontline and second line health care workers during the Coronavirus disease 2019 pandemic in Italy. JAMA Network Open, 3(5). https://dx.doi.org/10.1001\%2Fjamanetworkopen.2020.10185

Staats, P. Hekmat, H. \& Staats, A. (1996). The Psychological Behaviorism Theory of Pain: A Basis for Unity. $\begin{array}{llll}\text { Pain } & \text { Forum, } & 5 & \text { 194), }\end{array}$ https://www.researchgate.net/publication/323003596 The Psychological Behaviorism Theory of Pain A Basis for Unity

Tabernero, M. \& Politis, D. (2014). Reconocimiento de emociones básicas y complejas en la variante conductual de la demencia frontotemporal. Neurología Argentina, 5(2): 57-65. http://dx.doi.org/10.1016/i.neuarg.2012.12.004

Tabernero, C., Arenas, A., Cuadrado, E. \& Luque, B. (2014). Incertidumbre y orientación hacia los errores en tiempos de crisis. La importancia de generar confianza fomentando la eficacia colectiva. Papeles del Psicólogo, 35, (2), 107-114. https://www.redalyc.org/pdf/778/77831095009.pdf

Thielmann, K. \& Illnait, J. (2012). La crisis y la salud. ¿La salud en crisis? Revista Cubana de Salud Pública, 38(2), 278-285. $\quad$ http://scielo.sld.cu/scielo.php?script=sci arttext\&pid=S086434662012000200011\&lng=es\&tlng=es

Urzúa, A., Vera, P., Caqueo, A. \& Polanco, R. (2020). La Psicología en la prevención y manejo del COVID-19. Aportes desde la evidencia inicial. Terapia psicológica, 38(1), 103-118. https://dx.doi.org/10.4067/S0718-48082020000100103

Useche, O. (2008). Miedo, seguridad y resistencias: el miedo como articulación política de la negatividad. Polis, Revista de la Universidad Bolivariana, (19). https://www.redalyc.org/comocitar.oa?id=30501908 